Research Article

\title{
Reflection and Refraction of Shear Horizontal Waves in Peridynamic Medium
}

\author{
Xiaolong Zhang, ${ }^{1}$ Yaru Lu, ${ }^{2}$ Liyu Chen, ${ }^{3}$ and Zhenying Xu $\mathbb{D}^{1}$ \\ ${ }^{1}$ School of Mechanical Engineering, Jiangsu University, Zhenjiang 212013, China \\ ${ }^{2}$ Department of Life Science, Tangshan Normal University, Tangshan 063000, China \\ ${ }^{3}$ College of Mathematics and Computer Science, Tangshan Normal University, Tangshan 063000, China \\ Correspondence should be addressed to Zhenying Xu; zhyxu77@163.com
}

Received 15 September 2019; Revised 12 August 2020; Accepted 29 August 2020; Published 10 September 2020

Academic Editor: Said Elias

Copyright (c) 2020 Xiaolong Zhang et al. This is an open access article distributed under the Creative Commons Attribution License, which permits unrestricted use, distribution, and reproduction in any medium, provided the original work is properly cited.

\begin{abstract}
This work uses peridynamic theory to obtain the internal force density of a shear horizontal (SH) wave, which is only connected with shear modulus. We then established the reflection equation of a SH wave in peridynamic medium based on the force boundary condition of the $\mathrm{SH}$ wave at the virtual boundary layer. The $\mathrm{SH}$ wave reflection characteristic in peridynamic medium is similar to the results of traditional theory, which was verified by simulations and experiments in steel plates. The refraction characteristics of a SH wave in peridynamic medium were obtained based on the continuity of the force and the displacement at the refraction point. These features established the relationship between bonds and wave angle. The refraction and reflection characteristics of a $\mathrm{SH}$ wave in a peridynamic medium were also verified by numerical simulations and experiments in a welded structure.
\end{abstract}

\section{Introduction}

Large steel structures are widely used in daily life, and the safety of steel structures is very important [1]. Ultrasonic testing is widely used to inspect large steel structures because it is nondestructive and fast $[2,3]$. In order to detect defects in steel structures by using ultrasound, we need to study the propagation characteristics of ultrasound in steel structures. Ordinary steel structures are usually in the form of plates and welds $[4,5]$. Therefore, this work studies ultrasound reflection at the boundary and refraction at the interface of different components. In recent years, multiple studies on the wave propagation and their reflection and refraction behaviour have been inferred by Singh et al. $[6,7]$ and Guha et al. $[8,9]$.

In general, the finite element method [10-12] is used to solve the problem of ultrasonic propagation in plate and welded structures. In 2015, Kamas et al. [13] discussed the theoretical and experimental analysis of weld-guided waves as well as guided wave propagation. In 2016, Pau et al. [14] used the reciprocity method to study the interaction of $\mathrm{SH}_{0}$ mode with discontinuities in plate waveguides. In the same year, scattering of plane $\mathrm{SH}$ waves by a rectangular cavity embedded at shallow depth in an elastic half-space was investigated by Liu et al. [15]. In 2017, Achillopoulou and Pau [16] used a finite element model to investigate the interaction of shear and Lamb waves with different kinds of defects in plates. In 2019, Fan et al. [17] investigated the wave transmission and reflection of an elastic $\mathrm{P}$-wave at a single joint for normal incidence. However, the structures usually have defects, but the finite element method is a numerical solution of differential equations based on partial derivatives. The partial derivatives do not exist at the defects. Thus, the peridynamics method was used to solve this problem.

Peridynamics is an integral equation that does not require derivatives. In this paper, the peridynamic method was used to study the reflection of the ultrasonic wave at the boundary and the refraction between different structures. The theory of peridynamic wave was first proposed by Silling [18] in 2000, and the propagation of linear stress waves and 
the wave dispersion was investigated. In 2003, Silling et al. [19] discussed the wave propagation in the discontinuities bar. Zimmermann [20] later explored many characteristics of peridynamic theory, including wave motion, stability of peridynamic solution, and numerical solution techniques. The relationship between stress, displacement, and location under small deformation conditions of microelastic peridynamic equation was described by Silling et al. [21] in 2007. In 2010, Silling [22] proposed the linearization theory of peridynamics, and this theory makes the peridynamic wave equation easier to solve. In 2016, Bazant et al. [23] systematically studied the peridynamic stress wave and analyzed the bond-based peridynamic stress wave and dispersion. In the same year, Silling [24] studied the solitary waves in the peridynamic medium and found that the velocity of the solitary wave is greater than the elastic wave. In 2017, Butt et al. [25] associated the peridynamic nonlocality expressed by the horizon with a characteristic length scale related to the material microstructure. They compared the dispersion curves obtained from peridynamics with experimental data. In 2019, Zhang et al. [26] studied wave propagation and dispersion in the peridynamic medium and found that the $\mathrm{SH}$ wave also dispersed in an unbounded steel medium.

In this paper, because of the simple form of $\mathrm{SH}$, there is no waveform conversion when $\mathrm{SH}$ interacts with the boundary. We choose $\mathrm{SH}$ waves as the research object. By studying peridynamic properties, the relationship between the micromodulus and shear modulus in the SH wave was obtained by decomposing the force between the material points. The reflection and refraction characteristics of $\mathrm{SH}$ waves at the boundary were analyzed. We used simulation and experiments of the plate and welded structures to conclude that the reflection at the boundary of the weld seam is the main factor that forms an $\mathrm{SH}$-guided wave. The material difference between the weld seam and steel plate aggravates the formation of $\mathrm{SH}$-guided waves in the weld seam.

\section{SH Waves on Linear Peridynamics}

The linearized version of the peridynamic theory has been investigated previously $[19,22,27-29]$. The equation of motion in the linearized theory is given as follows:

$$
\rho(\mathbf{x}) \ddot{u}(\mathbf{x}, t)=\int_{N_{\mathbf{x}}} C(\mathbf{x}, \mathbf{q})(\mathbf{u}(\mathbf{q}, t)-\mathbf{u}(\mathbf{x}, t)) \mathrm{d} V_{\mathbf{q}}+\mathbf{b}(\mathbf{x}, t),
$$

where $\rho$ is the density; $\mathbf{u}$ is the displacement; $\mathbf{b}$ is the body force density; the integral term is the internal force density; and $\mathbf{x}$ is the position in the reference configuration; and $t$ is time. Term $N_{\mathbf{x}}$ is a neighborhood of $\mathbf{x}$ as discussed in [22]; the radius of $N_{\mathrm{x}}$ is, in general, $2 \delta$, where $\delta$ is the horizon. Terms $\mathbf{x}$ and $\mathbf{q}$ are material points in $N_{\mathbf{x}}$; $\mathbf{C}$ is a tensor-valued function called the micromodulus. When the material is a linear isotropic solid, the micromodulus $\mathbf{C}$ is defined as follows $[22,28]$ :

$$
\begin{aligned}
\mathbf{C}(\mathbf{x}, \mathbf{q})= & \left(\frac{15 \mu}{m^{2}}-\frac{9 \kappa}{m^{2}}\right) \int_{H_{\mathbf{p}}} \Phi(|\mathbf{p}-\mathbf{x}|) \oplus(|\mathbf{p}-\mathbf{q}|)(\mathbf{p}-\mathbf{x}) \\
& \otimes(\mathbf{p}-\mathbf{q}) \mathrm{d} V_{\mathbf{p}}+2 \gamma(\mathbf{q}-\mathbf{x}), \\
\mathbf{r}(\boldsymbol{\xi})= & \frac{15 \mu}{m} \Phi(|\xi|)(\mathbf{M} \otimes \mathbf{M}),
\end{aligned}
$$

where $\kappa$ is the bulk modulus, $\mu$ is the shear modulus, $\mathbf{M}=(\xi / \xi \mid), m=\int_{H} \omega(|\xi|)|\xi|^{2} \mathrm{~d} V_{\xi}, \boldsymbol{\omega}$ is the weight function, $\otimes$ is expressed as the Kronecker product, and $\xi=\mathbf{q}-\mathbf{x}$ is the bond between material points. Term $\mathbf{p}$ is the material point in the horizon of $\mathbf{x}$.

The micromodulus $\mathbf{C}$ of inviscid fluid is defined as follows [22]:

$$
\mathbf{C}(\mathbf{x}, \mathbf{q})=-\frac{9 \kappa}{m^{2}} \int_{H_{\mathbf{p}}} \bowtie(|\mathbf{p}-\mathbf{x}|) \oplus(|\mathbf{p}-\mathbf{q}|)(\mathbf{p}-\mathbf{x}) \otimes(\mathbf{p}-\mathbf{q}) \mathrm{d} V_{\mathbf{p}} .
$$

The liquid is not viscous, and thus it can only form longitudinal waves. When combined with (3), we can obtain the micromodulus, that is, only related to $\mathrm{SH}$ wave internal force density, in linear elastic solids:

$$
\begin{aligned}
\mathbf{C}(\mathbf{x}, \mathbf{q})= & \frac{15 \mu}{m^{2}} \int_{H_{\mathbf{p}}} \varpi(|\mathbf{p}-\mathbf{x}|) \oplus(|\mathbf{p}-\mathbf{q}|)(\mathbf{p}-\mathbf{x}) \\
& \otimes(\mathbf{p}-\mathbf{q}) \mathrm{d} V_{\mathbf{p}}+2 \gamma(\mathbf{q}-\mathbf{x}) .
\end{aligned}
$$

When the SH wave propagates on the surface $x_{1} o x_{2}$, the direction of force is along $x_{3}$. At this time, the internal force density along the $x_{3}$ is as follows:

$$
\begin{aligned}
\tau_{3}= & \int_{N_{\mathbf{x}}} c_{31}\left(u_{1}(\mathbf{q}, t)-u_{1}(\mathbf{x}, t)\right)+c_{32}\left(u_{2}(\mathbf{q}, t)-u_{2}(\mathbf{x}, t)\right) \\
& +c_{33}\left(u_{3}(\mathbf{q}, t)-u_{3}(\mathbf{x}, t)\right) \mathrm{d} V_{\mathbf{q}} .
\end{aligned}
$$

Here, $c_{i j}$ are components of $\mathbf{C}(\mathbf{x}, \mathbf{q})$. Since the $\mathrm{SH}$ wave only has a displacement along $x_{3}$, formula (5) is reduced to the following:

$$
\tau_{3}=\int_{N_{\mathbf{x}}} c_{33}\left(u_{3}(\mathbf{q}, t)-u_{3}(\mathbf{x}, t)\right) \mathrm{d} V_{\mathbf{q}} .
$$

Therefore, the internal force density of SH wave is only affected by the micromodulus $c_{33}$, and as $\mathrm{SH}$ wave is a plane wave, the material points $\mathbf{q}$ and $\mathbf{x}$ in formula (4) are in the same plane $x_{1} o x_{2}$; thus, $\gamma_{33}=0$, which is the component of $\gamma$ in (4). Therefore, the elastic micromodulus matrix can be simplified to the following:

$$
c_{i j}(\mathbf{x}, \mathbf{q})=\frac{15 \mu}{m^{2}} \int_{H_{p}} \omega(|\mathbf{p}-\mathbf{x}|) \oplus(|\mathbf{p}-\mathbf{q}|)\left(p_{i}-x_{i}\right) \otimes\left(p_{j}-q_{j}\right) \mathrm{d} V_{\mathbf{p}} .
$$

Since the weighted volume $m$ and the bonds in (7) are only related to the geometric subdivision, the propagation of 
SH waves in different materials is only related to the shear modulus $\mu$.

\section{The SH Waves Reflection in Peridynamic Medium}

3.1. The Reflection Theory of $\mathrm{SH}$ Waves. In Figure $1, x_{2}=0$ is the interface of a homogeneous solid material and air. The lower part is a homogeneous solid material, and the upper part is air. The SH wave cannot propagate in the air, and no wave transforms at the interface. Thus, there are only reflected $\mathrm{SH}$ waves at the interface. Given a beam of incident waves $p_{0}$, the angle between the incident wave and the normal line $x_{1}=0$ is $\theta_{0}$. The reflected wave $p_{2}$ is obtained through the reflection interface $x_{2}=0$, and the angle between the reflected wave and the normal line is $\theta_{2}$. Terms $\delta_{1}$ and $\delta_{2}$ are the radius of any horizon in peridynamics.

Given the incident wave with the amplitude $A_{0}$, wavenumber $k_{0}$, and velocity $c_{T}$, we can obtain the incident wave function as

$$
u_{3}^{(0)}=A_{0} \exp \left[i k_{0}\left(x_{1} \sin \theta_{0}+x_{2} \cos \theta_{0}-c_{T} t\right)\right] .
$$

Similarly, assuming that the amplitude is $A_{2}$, the wavenumber is $k_{2}$, and the velocity is $c_{T}$, the wave function of the reflected wave is

$$
u_{3}^{(2)}=A_{2} \exp \left[i k_{2}\left(x_{1} \sin \theta_{2}-x_{2} \cos \theta_{2}-c_{T} t\right)\right] .
$$

In classical mechanics, $\mathrm{SH}$ wave reflection problems are usually divided into two kinds: fixed boundary and free boundary. A fixed boundary requires the sum of displacement vectors to be zero. Under the theory of peridynamics, the analytical method of SH waves is the same as in classical theory. This paper does not do further analysis. For a free boundary, the force density should be zero at the boundary. The peridynamic method is quite different from traditional mechanics because the internal force in the classical method is different from peridynamics.

In this section, the reflection of $\mathrm{SH}$ waves based on peridynamics is introduced in detail. The SH wave produces two internal force densities when reflecting at the free boundary. One is generated by the incident wave, and the other is generated by the reflecting wave. In the peridynamic method, the boundary conditions are applied to the virtual boundary layer [30], as shown in Figure 1. Term q shows material points on the virtual layer adjacent to the reflection point. Terms $\mathbf{q}_{0}$ and $\mathbf{q}_{2}$ are the material points on the incident region and the reflected region, respectively (Figure 1). From the peridynamic method, we can obtain two internal force density values:

$$
\begin{aligned}
& \tau_{3}^{(0)}=\int_{N_{0}} \mathbf{C}\left(\mathbf{q}, \mathbf{q}_{0}\right)\left(u_{3}^{(0)}\left(\mathbf{q}_{0}, t\right)-u_{3}^{(0)}(\mathbf{q}, t)\right) \mathrm{d} V_{\mathbf{q}_{0}}, \\
& \tau_{3}^{(2)}=\int_{N_{2}} \mathbf{C}\left(\mathbf{q}, \mathbf{q}_{2}\right)\left(u_{3}^{(2)}\left(\mathbf{q}_{2}, t\right)-u_{3}^{(2)}(\mathbf{q}, t)\right) \mathrm{d} V_{\mathbf{q}_{2}} .
\end{aligned}
$$

Here, $\mathbf{C}\left(\mathbf{q}, \mathbf{q}_{0}\right)$ and $\mathbf{C}\left(\mathbf{q}, \mathbf{q}_{2}\right)$ are the micromodulus of materials on the incident and reflected regions, respectively. Terms $\mathrm{N}_{0}$ and $\mathrm{N}_{2}$ correspond to the horizon, and the

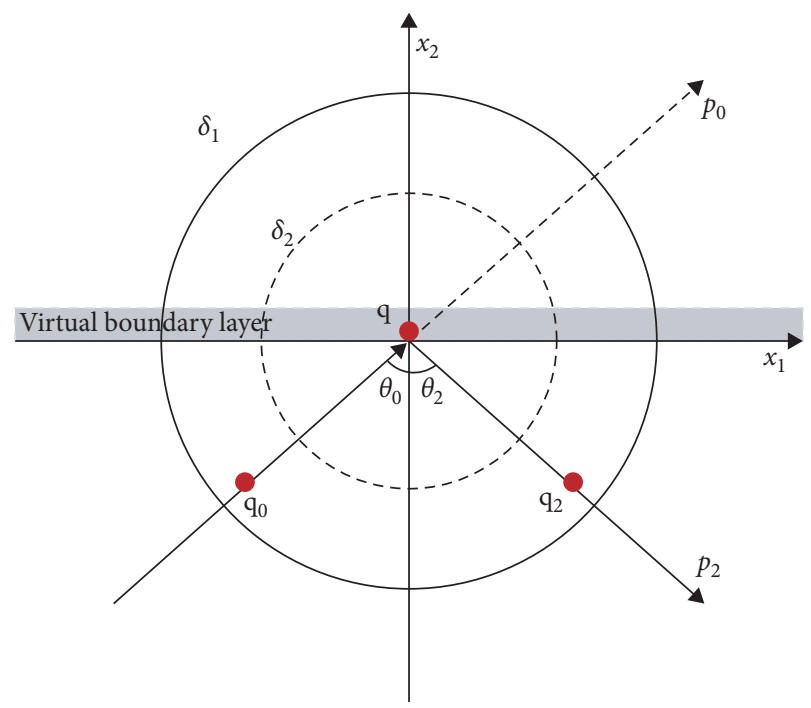

FIGURE 1: SH wave reflection.

radius of the horizon is equal. $\tau_{3}^{(0)}$ and $\tau_{3}^{(2)}$ correspond to the internal force density of the incident and reflected waves near the boundary of the virtual layer. Based on peridynamic theory, the internal force density at the virtual layer is $\tau_{3}^{(0)}+\tau_{3}^{(2)}=0$ [30]. Thus, from (5), we can obtain the following:

$$
\begin{aligned}
& \int_{N_{0}} c_{33}\left(\mathbf{q}, \mathbf{q}_{0}\right)\left(u_{3}^{(0)}\left(\mathbf{q}_{0}, t\right)-u_{3}^{(0)}(\mathbf{q}, t)\right) \mathrm{d} V_{\mathbf{q}_{0}} \\
& \quad+\int_{N_{2}} c_{33}\left(\mathbf{q}, \mathbf{q}_{2}\right)\left(u_{3}^{(2)}\left(\mathbf{q}_{2}, t\right)-u_{3}^{(2)}(\mathbf{q}, t)\right) \mathrm{d} V_{\mathbf{q}_{2}}=0 .
\end{aligned}
$$

Under the numerical discretization, (11) can be written as

$$
\begin{aligned}
& \sum c_{33}\left(\mathbf{q}, \mathbf{q}_{0}\right)\left(u_{3}^{(0)}\left(\mathbf{q}_{0}, t\right)-u_{3}^{(0)}(\mathbf{q}, t)\right) \Delta V_{\mathbf{q}_{0}} \\
& \quad+\sum c_{33}\left(\mathbf{q}, \mathbf{q}_{2}\right)\left(u_{3}^{(2)}\left(\mathbf{q}_{2}, t\right)-u_{3}^{(2)}(\mathbf{q}, t)\right) \Delta V_{\mathbf{q}_{2}}=0 .
\end{aligned}
$$

The stress equilibrium conditions (11) and (12) are valid when the radius of the peridynamic horizon is arbitrary. If the horizon only contains two material points, i.e., an incident material point $\mathbf{q}_{0}^{1}$ and a reflective material point $\mathbf{q}_{2}^{1}$, then we can obtain

$$
\begin{aligned}
& c_{33}\left(\mathbf{q}, \mathbf{q}_{0}^{(\mathbf{1})}\right)\left(u_{3}^{(0)}\left(\mathbf{q}_{0}^{(\mathbf{1})}, t\right)-u_{3}^{(0)}(\mathbf{q}, t)\right) \Delta V_{\mathbf{q}_{0}^{(1)}} \\
& \quad+c_{33}\left(\mathbf{q}, \mathbf{q}_{2}^{(\mathbf{1})}\right)\left(u_{3}^{(2)}\left(\mathbf{q}_{2}^{(\mathbf{1})}, t\right)-u_{3}^{(2)}(\mathbf{q}, t)\right) \Delta V_{\mathbf{q}_{2}^{(1)}}=0 .
\end{aligned}
$$

The weight function in (7) is only related to the length of the bond, and the material points $\mathbf{q}_{0}^{(1)}$ and $\mathbf{q}_{2}^{(1)}$ are symmetric about $x_{1}=0$. Thus, micromodulus can be determined by (7) to be

$$
c_{33}\left(\mathbf{q}, \mathbf{q}_{0}^{(\mathbf{1})}\right)=c_{33}\left(\mathbf{q}, \mathbf{q}_{2}^{(\mathbf{1})}\right) .
$$

The subdivision of the incident and reflected waves is the same: 


$$
\Delta V_{\mathbf{q}_{0}^{(1)}}=\Delta V_{\mathbf{q}_{2}^{(1)}} .
$$

From (13) to (15), we conclude that

$u_{3}^{(0)}\left(\mathbf{q}_{0}^{(1)}, t\right)-u_{3}^{(0)}(\mathbf{q}, t)+u_{3}^{(2)}\left(\mathbf{q}_{2}^{(1)}, t\right)-u_{3}^{(2)}(\mathbf{q}, t)=0$.

Similarly, when there are four material points in a horizon, by combining (12) with (14)-(16), we derive the following:

$$
u_{3}^{(0)}\left(\mathbf{q}_{0}^{(2)}, t\right)-u_{3}^{(0)}(\mathbf{q}, t)+u_{3}^{(2)}\left(\mathbf{q}_{2}^{(2)}, t\right)-u_{3}^{(2)}(\mathbf{q}, t)=0 .
$$

By analogy, we can get material points that are symmetric about $x_{1}=0$ in the horizon domain:

$$
u_{3}^{(0)}\left(\mathbf{q}_{0}, t\right)-u_{3}^{(0)}(\mathbf{q}, t)+u_{3}^{(2)}\left(\mathbf{q}_{2}, t\right)-u_{3}^{(2)}(\mathbf{q}, t)=0 .
$$

For the same bond length,

$$
|\xi|=\left|\mathbf{q}_{0}-\mathbf{q}\right|=\left|\mathbf{q}_{2}-\mathbf{q}\right| \text {. }
$$

Substituting (8) and (9) into (18), we obtain

$$
\begin{aligned}
& A_{0} \exp \left[i k_{0}\left(x_{1}^{\left(q_{0}\right)} \sin \theta_{0}+x_{2}^{\left(q_{0}\right)} \cos \theta_{0}-c_{T} t\right)\right] \\
& \quad-A_{0} \exp \left[i k_{0}\left(x_{1}^{(q)} \sin \theta_{0}+x_{2}^{(q)} \cos \theta_{0}-c_{T} t\right)\right] \\
& \quad+A_{2} \exp \left[i k_{2}\left(x_{1}^{\left(q_{2}\right)} \sin \theta_{2}-x_{2}^{\left(q_{2}\right)} \cos \theta_{2}-c_{T} t\right)\right] \\
& \quad-A_{2} \exp \left[i k_{2}\left(x_{1}^{(q)} \sin \theta_{2}-x_{1}^{(q)} \cos \theta_{2}-c_{T} t\right)\right]=0 .
\end{aligned}
$$

From (19), we can conclude that

$$
\begin{aligned}
& x_{1}^{\left(\mathbf{q}_{0}\right)}-x_{1}^{(\mathbf{q})}=-|\xi| \sin \theta_{0}, \\
& x_{2}^{\left(\mathbf{q}_{0}\right)}-x_{2}^{(\mathbf{q})}=-|\xi| \cos \theta_{0}, \\
& x_{1}^{\left(\mathbf{q}_{2}\right)}-x_{1}^{(\mathbf{q})}=|\xi| \sin \theta_{2}, \\
& x_{2}^{\left(\mathbf{q}_{2}\right)}-x_{2}^{(\mathbf{q})}=-|\xi| \cos \theta_{2} .
\end{aligned}
$$

Substituting (21) and (22) into (20), we obtain

$$
\begin{aligned}
& A_{0}\left(\exp \left(-i k_{0}|\xi|\right)-1\right) \exp \left[i k_{0}\left(x_{1}^{(\mathbf{q})} \sin \theta_{0}+x_{2}^{(\mathbf{q})} \cos \theta_{0}-c_{T} t\right)\right] \\
& \quad+A_{2}\left(\exp \left(i k_{2}|\xi|\right)-1\right) \exp \left[i k_{2}\left(x_{1}^{(\mathbf{q})} \sin \theta_{2}-x_{2}^{(\mathbf{q})} \cos \theta_{2}-c_{T} t\right)\right]=0 .
\end{aligned}
$$

When the radius of the horizon is given as $\delta \longrightarrow 0$, then under the Taylor expansion, (23) can be written as

$$
\begin{aligned}
& A_{0}\left(-i k_{0}|\xi|\right) \exp \left[i k_{0}\left(x_{1}^{(\mathbf{q})} \sin \theta_{0}+x_{2}^{(\mathbf{q})} \cos \theta_{0}-c_{T} t\right)\right] \\
& \quad+A_{2}\left(i k_{2}|\xi|\right) \exp \left[i k_{2}\left(x_{1}^{(\mathbf{q})} \sin \theta_{2}-x_{2}^{(\mathbf{q})} \cos \theta_{2}-c_{T} t\right)\right]=0 .
\end{aligned}
$$

The interface is $x_{2}=0$. When substituted into (24), the equation can be recast as

$$
\begin{aligned}
& A_{0} k_{0} \exp \left[i k_{0}\left(x_{1}^{(\mathbf{q})} \sin \theta_{0}-c_{T} t\right)\right] \\
& \quad=A_{2} k_{2} \exp \left[i k_{2}\left(x_{1}^{(\mathbf{q})} \sin \theta_{2}-c_{T} t\right)\right] .
\end{aligned}
$$

From (25), we conclude the following:

$$
\begin{aligned}
A_{0} & =A_{2}, \\
k_{0} & =k_{2}, \\
\theta_{0} & =\theta_{2} .
\end{aligned}
$$
[31].

This result is the same as that obtained by classical theory

3.2. SH Wave Propagate in Steel Plate. The reflection characteristics of the $\mathrm{SH}$ wave were studied with a steel plate. The size of the steel plate is $1610 \times 600 \times 6 \mathrm{~mm}^{3}$, which is the same as the welded model presented in Section 3.3. The selected steel plate material density is $7932 \mathrm{~kg} / \mathrm{m}^{3}$, the modulus of elasticity is $210 \mathrm{GPa}$, Poisson's ratio is 0.3 , and the $\mathrm{SH}$ wave velocity is $3191 \mathrm{~m} / \mathrm{s}$. In order to examine the propagation characteristics of the $\mathrm{SH}$-guided wave in the steel plate, sensors are set in the steel plate. The distribution of sensors and load is shown in Figure 2.

Of these, $\mathrm{B}$ and $\mathrm{C}$ are the midpoint in the length direction of the steel plate and are on the edge of the steel plate. The surface force load in direction $x_{3}$ is applied to point $\mathrm{D}$. This will generate an $\mathrm{SH}$ wave that propagates along the line $\mathrm{BC}$ with the vibration in direction $x_{3}$. Sensor $\mathrm{E}$ is located in the middle of the steel plate and on the $\mathrm{BC}$ line $30 \mathrm{~mm}$ from point $\mathrm{B}$; Sensor F is $15 \mathrm{~mm}$ from the $\mathrm{BC}$ line and $30 \mathrm{~mm}$ from the vertical edge of the steel plate. Sensor G is $25 \mathrm{~mm}$ horizontal from the BC line and $30 \mathrm{~mm}$ vertical from the edge of the steel plate. Sensor $\mathrm{H}$ is $35 \mathrm{~mm}$ horizontal from the $\mathrm{BC}$ line and $30 \mathrm{~mm}$ vertical from the edge of the steel plate.

A 5-period Hans window-modulated sinusoidal wave function was applied to point D in Figure 2, and the wave function of the load is as follows:

$$
\sigma=A\left[1-\cos \left(\frac{2 \pi \mathrm{ft}}{n}\right)\right] \cdot \sin (2 \pi \mathrm{ft}),
$$

where the amplitude is $A=10 \mathrm{kPa}$, the frequency is $n=5$, and the number of periods is $n=5$.

In order to study the propagation of the $\mathrm{SH}$ wave in the steel plate, the $\mathrm{SH}$-guided wave in the steel plate is numerically simulated. The iteration step is $10^{-7} \mathrm{~s}$, and the simulation time is $1 \mathrm{~ms}$; the peridynamic subdivision is $h=2 \mathrm{~mm}$ [32], the weight function is $\delta=3.1 \mathrm{~h}$, and the horizon radius is $\delta=3.1 \mathrm{~h}$. The signal in the sensor is obtained, as shown in Figure 3.

Figure 3 shows that signal 1 on sensor E was generated by an initial signal. The amplitude is larger because sensor $\mathrm{E}$ is located on the midline BC and is close to the source. Sensors F, G, and $\mathrm{H}$ are far from the midline $\mathrm{BC}$, and the amplitude of signal 1 decreases gradually. This phenomenon shows that the $\mathrm{SH}$-guided waves produced by shear load at point $\mathrm{D}$ are mainly propagating along the $\mathrm{BC}$ direction, which agrees with the results of traditional dynamic. Signal 2 is the initial signal propagated to the B end and is then reflected. Signal 2 appears later than signal 1 and becomes dispersed. Signal 1 is 


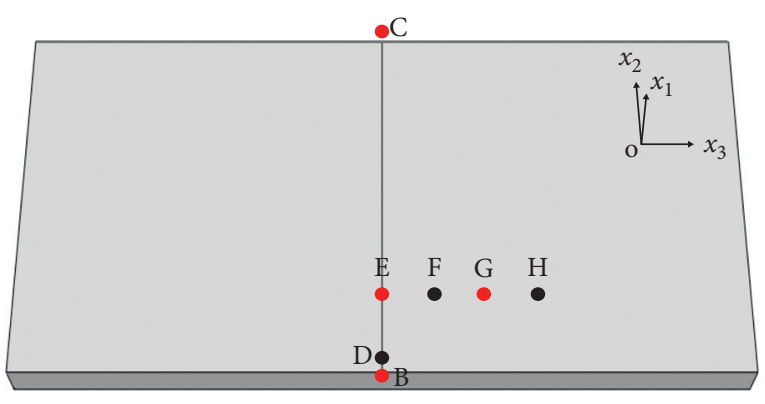

Figure 2: Sensor and load distribution on the steel plate.
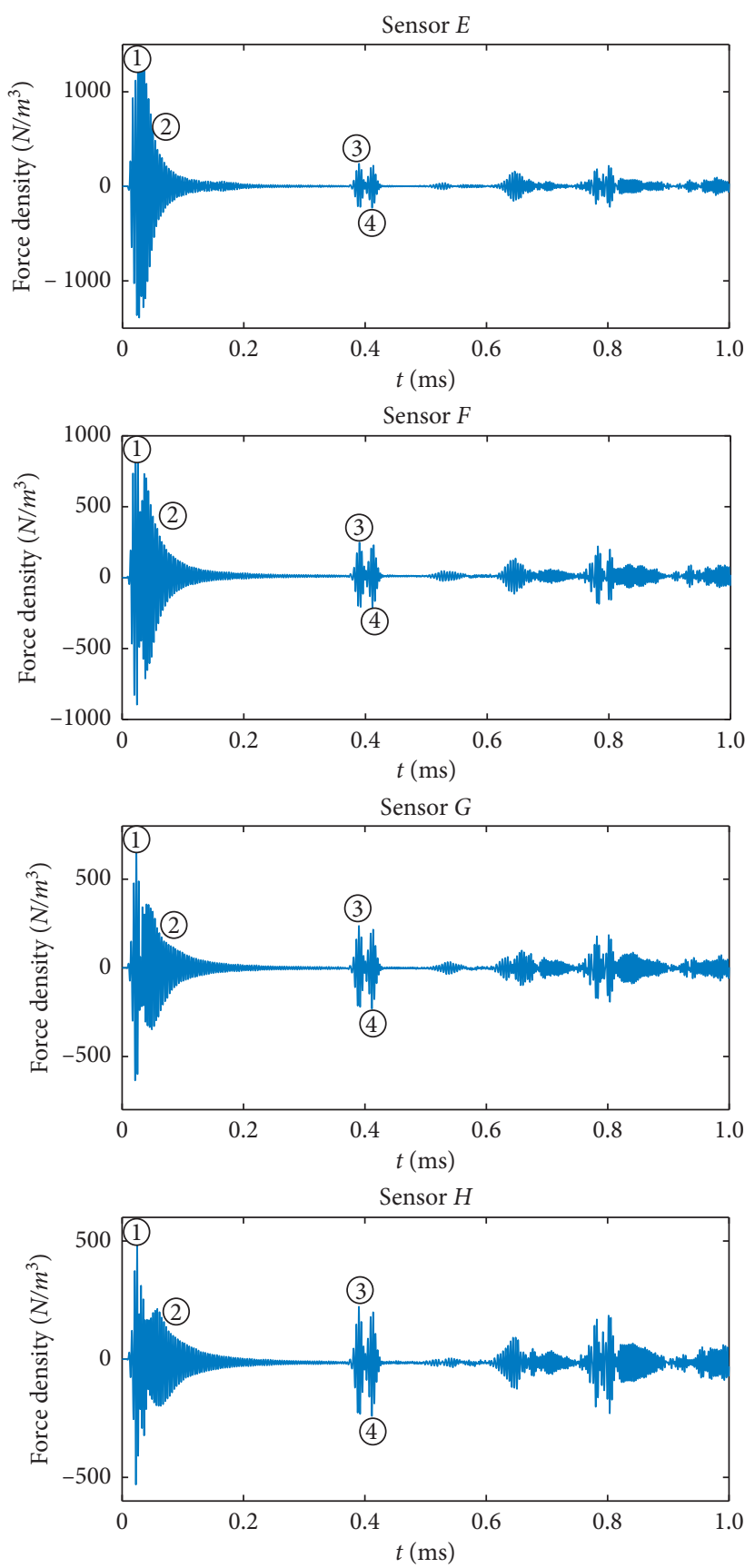

FigURE 3: Sensor signal on the steel plate by numerical simulation. reflected by $\mathrm{C}$ and ends the generated signal 3. Similarly, the reflected signal 3 also decreases as the sensor moves away from the midline. Signal 4 is generated by signal 2 and is reflected by the $C$ end. Signal 1 is the initial signal close to the source, and thus it might disturb the signal source. Thus, signals 2 and 4 were obviously dispersed. Here, signal 3 was analyzed further. Specific data from signal 3 are shown in Table 1 .

Table 1 shows that the peak time of signal 3 is gradually delayed because the positions of sensors F, G, and $H$ are far from the center line. Compared with the peak data, the amplitude of signal 3 on sensor $\mathrm{E}$ is not obvious because the $\mathrm{SH}$-guided wave radiates more energy to the outside during propagation. The amplitudes of sensors F, G, and $\mathrm{H}$ far from the midline gradually decrease, and the peak time of signal 3 is delayed. The radiation rate of the guided wave is defined to study the radiation energy of the guided wave. Selecting the maximum amplitude of signal 3 in sensors $\mathrm{E}$ and $\mathrm{F}$ to define the guided wave emissivity at point $F$, we obtain the following:

$$
\eta=\frac{A_{F}}{A_{E}+A_{F}} .
$$

Here, $\eta$ is the radiation rate of $\mathrm{SH}$-guided waves from the weld seam to the steel plate, $A_{E}$ is a peak of signal 3 in sensor $\mathrm{E}$, and $A_{F}$ is a peak of signal 3 in sensor F. Therefore, the radiation rate can be obtained by (28) to be $50.8 \%$.

3.3. Steel Plate Experiment. The steel plate has the same size and material parameters as the simulation. The load excited by the $\mathrm{SH}$ wave piezoelectric (PZT) sensor at point $\mathrm{D}$ is shown in Figure 2. The SH wave PZT sensor is applied at position E (Figure 2) to receive the $\mathrm{SH}$ wave, and the electrical signals are measured by an oscilloscope [33]. The $\mathrm{SH}$-guided wave among the plate structure mainly propagates in the direction perpendicular to vibration. There is clutter interference in the experiment. The signals of sensors F, G, and $\mathrm{H}$ are not obvious, and detailed analysis has not been carried out in this paper. Only sensor E has an obvious signal (Figure 4).

Figure 4 shows that the signal on the oscilloscope is basically the same as the signal of the sensor in Figure 3. However, due to the randomness of the signal collected by the oscilloscope, the signal on the oscilloscope has a delay of $0.4 \mathrm{~ms}$. The scattering of the $\mathrm{SH}$ wave by grains results in a $0.016 \mathrm{~V}$ clutter, which intensifies the dispersion of the $\mathrm{SH}$ wave. The amplitude of signal 3 is $0.04 \mathrm{~V}$ at $0.819 \mathrm{~ms}$. The time increases compared with simulation. Through the comparison of peridynamic numerical simulation and experiment signal, we see that the peridynamic theory can well reflect the reflection characteristics of the $\mathrm{SH}$ wave in the steel plate.

\section{The SH Waves Refraction in Peridynamic Medium}

4.1. The Theory ofSH Waves by Refraction. In Figure 5, $x_{2}=0$ is the interface of two different materials. The shear modulus of the lower part is $\mu_{0}$ and the upper part is $\mu_{4}$. Given an 
TABle 1: The data of signal 3 in the steel plate.

\begin{tabular}{lcccc}
\hline & Sensor E & Sensor F & Sensor G & Sensor H \\
\hline Time $(\mathrm{ms})$ & 0.3900 & 0.3900 & 0.3901 & 0.3902 \\
Amplitude $\left(\mathrm{N} / \mathrm{m}^{3}\right)$ & 240.0 & 248.0 & 237.0 & 222.2 \\
\hline
\end{tabular}

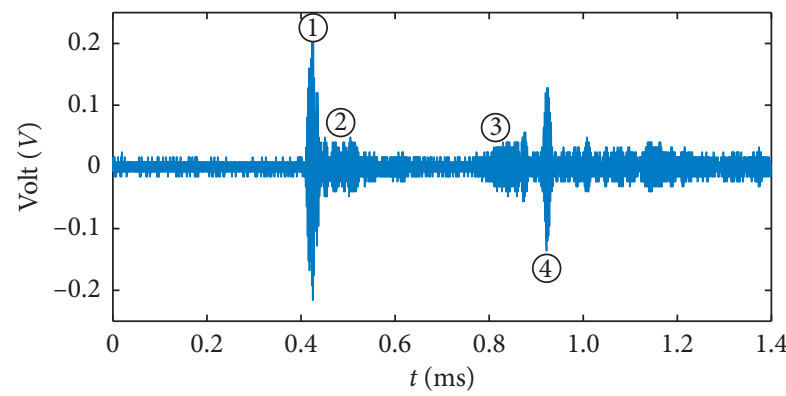

Figure 4: Oscilloscope signal of sensor E on the steel plate.

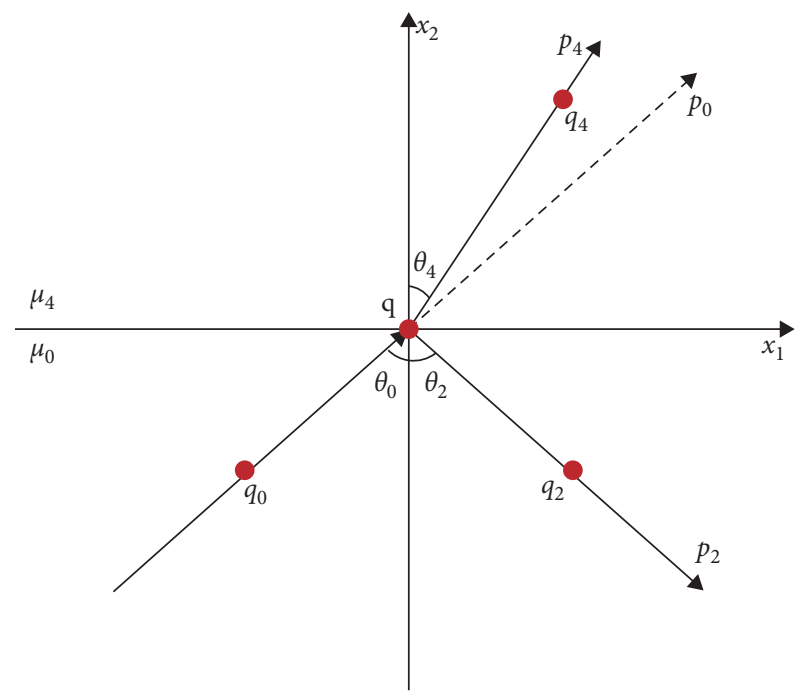

FIGURE 5: Refraction diagram of the SH wave.

incident wave along $p_{0}$, the angle between the incident wave and the normal line is $\theta_{0}$. The reflected wave $p_{2}$ is obtained through the reflection interface, and the angle between the reflected wave and the normal line is $\theta_{2}$. The refraction wave produced by the interface is $p_{4}$, and the refraction angle is $\theta_{4}$.

Assuming that the amplitude is $A_{4}$, the wave number is $k_{4}$, and velocity is $c_{T_{4}}$, the wave function of the refracted wave is as follows:

$$
u_{3}^{4}=A_{4} \exp \left[i k_{4}\left(x_{1} \sin \theta_{4}+x_{2} \cos \theta_{4}-c_{T_{4}} t\right)\right] .
$$

The wave function of incident and reflected waves can be calculated using (8) and (9).

From the continuity of displacement and stress of the $\mathrm{SH}$ wave of refraction point $\mathbf{q}$ in Figure 5, we obtain

$$
\begin{aligned}
& u_{3}^{(0)}+u_{3}^{(2)}=u_{3}^{(4)}, \\
& \tau^{(0)}+\tau^{(2)}=\tau^{(4)} .
\end{aligned}
$$

Substituting (8), (9), and (29) into (30) results in

$$
\begin{aligned}
& A_{0} \exp \left[i k_{0}\left(x_{1} \sin \theta_{0}-c_{T} t\right)\right]+A_{2} \exp \left[i k_{2}\left(x_{1} \sin \theta_{2}-c_{T} t\right)\right] \\
& \quad=A_{4} \exp \left[i k_{4}\left(x_{1} \sin \theta_{4}-c_{T_{4}} t\right)\right] .
\end{aligned}
$$

Since (32) contains exponents, the exponents should have the same size $u_{3}^{\prime}$, and the equations to be established are as follows:

$$
\begin{aligned}
u_{3}^{(0)} & =A_{0} u_{3}^{\prime}, \\
u_{3}^{(2)} & =A_{2} u_{3}^{\prime}, \\
u_{3}^{(4)} & =A_{4} u_{3}^{\prime}, \\
A_{0}+A_{2} & =A_{4}, \\
k_{0} \sin \theta_{0} & =k_{2} \sin \theta_{2}=k_{4} \sin \theta_{4}, \\
k_{0} c_{T} & =k_{2} c_{T}=k_{4} c_{T_{4}} .
\end{aligned}
$$

From (34) to (36), we can derive

$$
\begin{aligned}
k_{0} & =k_{2}, \\
\theta_{0} & =\theta_{2}, \\
k_{0}\left(\frac{c_{T}}{c_{T_{4}}}\right) & =k_{4}, \\
\sin \theta_{0} & =\left(\frac{c_{T}}{c_{T_{4}}}\right) \sin \theta_{4} .
\end{aligned}
$$

From stress equilibrium condition (31) and single direction stress in the $\mathrm{SH}$ wave, (31) is recast as

$$
\tau_{3}^{(0)}+\tau_{3}^{(2)}=\tau_{3}^{(4)} .
$$

Expansion of (38) results in

$$
\begin{aligned}
& \int_{N_{0}} c_{33}\left(\mathbf{q}, \mathbf{q}_{0}\right)\left(u_{3}^{(0)}\left(\mathbf{q}_{0}, t\right)-u_{3}^{(0)}(\mathbf{q}, t)\right) \mathrm{d} V_{\mathbf{q}_{0}} \\
& \quad+\int_{N_{2}} c_{33}\left(\mathbf{q}, \mathbf{q}_{2}\right)\left(u_{3}^{(2)}\left(\mathbf{q}_{2}, t\right)-u_{3}^{(2)}(\mathbf{q}, t)\right) \mathrm{d} V_{\mathbf{q}_{2}} \\
& =\int_{N_{4}} c_{33}\left(\mathbf{q}, \mathbf{q}_{4}\right)\left(u_{3}^{(4)}\left(\mathbf{q}_{4}, t\right)-u_{3}^{(4)}(\mathbf{q}, t)\right) \mathrm{d} V_{\mathbf{q}_{4}} .
\end{aligned}
$$

Here, $N_{4}$ is the horizon of refraction wave, and the refraction wave horizon is the same as that of the incident wave and reflected wave horizon. In this horizon, $c_{33}\left(\mathbf{q}, \mathbf{q}_{4}\right)$ is the micromodulus, and $\mathbf{q}_{4}$ is the material points (Figure 5). The subdivision of the material points is consistent with the region of the incident and reflected waves. With numerical discretization, (39) becomes 


$$
\begin{aligned}
& \sum c_{33}\left(\mathbf{q}, \mathbf{q}_{0}\right)\left(u_{3}^{(0)}\left(\mathbf{q}_{0}, t\right)-u_{3}^{(0)}(\mathbf{q}, t)\right) \Delta V_{\mathbf{q}_{0}} \\
& \quad+\sum c_{33}\left(\mathbf{q}, \mathbf{q}_{2}\right)\left(u_{3}^{(2)}\left(\mathbf{q}_{2}, t\right)-u_{3}^{(2)}(\mathbf{q}, t)\right) \Delta V_{\mathbf{q}_{2}} \\
& =\sum c_{33}\left(\mathbf{q}, \mathbf{q}_{4}\right)\left(u_{3}^{(4)}\left(\mathbf{q}_{4}, t\right)-u_{3}^{(4)}(\mathbf{q}, t)\right) \Delta V_{\mathbf{q}_{4}} .
\end{aligned}
$$

Similarly, (40) can yield

$$
\begin{aligned}
c_{33} & \left(\mathbf{q}, \mathbf{q}_{0}\right)\left(u_{3}^{(0)}\left(\mathbf{q}_{0}, t\right)-u_{3}^{(0)}(\mathbf{q}, t)\right) \\
& +c_{33}\left(\mathbf{q}, \mathbf{q}_{2}\right)\left(u_{3}^{(2)}\left(\mathbf{q}_{2}, t\right)-u_{3}^{(2)}(\mathbf{q}, t)\right) \\
& =c_{33}\left(\mathbf{q}, \mathbf{q}_{4}\right)\left(u_{3}^{(4)}\left(\mathbf{q}_{4}, t\right)-u_{3}^{(4)}(\mathbf{q}, t)\right) .
\end{aligned}
$$

Based on (7), the relationship between the material parameters in the reflected and incident region can be obtained as follows:

$$
c_{33}\left(\mathbf{q}, \mathbf{q}_{0}\right)=c_{33}\left(\mathbf{q}, \mathbf{q}_{2}\right) .
$$

Substituting (42) into (41) results in

$$
\begin{aligned}
c_{33} & \left(\mathbf{q}, \mathbf{q}_{0}\right)\left(u_{3}^{(0)}\left(\mathbf{q}_{0}, t\right)-u_{3}^{(0)}(\mathbf{q}, t)\right) \\
& +c_{33}\left(\mathbf{q}, \mathbf{q}_{0}\right)\left(u_{3}^{(2)}\left(\mathbf{q}_{2}, t\right)-u_{3}^{(2)}(\mathbf{q}, t)\right) \\
& =c_{33}\left(\mathbf{q}, \mathbf{q}_{4}\right)\left(u_{3}^{(4)}\left(\mathbf{q}_{4}, t\right)-u_{3}^{(4)}(\mathbf{q}, t)\right) .
\end{aligned}
$$

Then, substituting (33) into (43) results in

$$
c_{33}\left(\mathbf{q}, \mathbf{q}_{0}\right) A_{0}\left(\exp \left(-i k_{0}|\xi|\right)-1\right) u_{3}^{\prime}(\mathbf{q}, t)+c_{33}\left(\mathbf{q}, \mathbf{q}_{0}\right) A_{2}\left(\exp \left(i k_{2}|\xi|\right)-1\right) u_{3}^{\prime}(\mathbf{q}, t)
$$

Simplification of (44) yields

$$
c_{33}\left(\mathbf{q}, \mathbf{q}_{0}\right) A_{0}\left(\exp \left(-i k_{0}|\xi|\right)-1\right)+c_{33}\left(\mathbf{q}, \mathbf{q}_{0}\right) A_{2}\left(\exp \left(i k_{2}|\xi|\right)-1\right)=c_{33}\left(\mathbf{q}, \mathbf{q}_{4}\right) A_{4}\left(\exp \left(i k_{4}|\xi|\right)-1\right)
$$

When the radius of a given horizon tends to zero, the Taylor expansion yields

$$
c_{33}\left(\mathbf{q}, \mathbf{q}_{0}\right) A_{0}\left(-i k_{0}|\xi|\right)+c_{33}\left(\mathbf{q}, \mathbf{q}_{0}\right) A_{2}\left(i k_{2}|\xi|\right)=c_{33}\left(\mathbf{q}, \mathbf{q}_{4}\right) A_{4}\left(i k_{4}|\xi|\right) .
$$

With a combination of (34)-(36), (46) can be written as

$$
c_{33}\left(\mathbf{q}, \mathbf{q}_{0}\right) A_{0}-c_{33}\left(\mathbf{q}, \mathbf{q}_{0}\right) A_{2}=-c_{33}\left(\mathbf{q}, \mathbf{q}_{4}\right) A_{4}\left(\frac{c_{T}}{c_{T_{4}}}\right) .
$$

This equation is similar to (48) in classical theory [31]:

$$
\mu_{0} \cos \theta_{0} A_{0}-\mu_{0} \cos \theta_{0} A_{2}=\mu_{4} \cos \theta_{4} A_{4}\left(\frac{c_{T}}{c_{T_{4}}}\right) .
$$

Combined with the traditional elastic theory, the relationship between bonds and wave angle is as follows:

$$
\frac{c_{33}\left(\mathbf{q}, \mathbf{q}_{0}\right)}{\mu_{0} \cos \theta_{0}}=\frac{-c_{33}\left(\mathbf{q}, \mathbf{q}_{4}\right)}{\mu_{4} \cos \theta_{4}} .
$$

The amplitude ratios of reflected and refracted waves to incident waves can be obtained from (34) and (47):

$$
\begin{aligned}
& \frac{A_{2}}{A_{0}}=\frac{c_{T_{4}} c_{33}\left(\mathbf{q}, \mathbf{q}_{0}\right)+c_{T} c_{33}\left(\mathbf{q}, \mathbf{q}_{4}\right)}{c_{T_{4}} c_{33}\left(\mathbf{q}, \mathbf{q}_{0}\right)-c_{T} c_{33}\left(\mathbf{q}, \mathbf{q}_{4}\right)}, \\
& \frac{A_{4}}{A_{0}}=\frac{2 c_{T_{4}} c_{33}\left(\mathbf{q}, \mathbf{q}_{0}\right)}{c_{T_{4}} c_{33}\left(\mathbf{q}, \mathbf{q}_{0}\right)-c_{T} c_{33}\left(\mathbf{q}, \mathbf{q}_{4}\right)} .
\end{aligned}
$$

The results of (50) and (51) in peridynamic medium agree with those obtained by traditional theory [31].
4.2. SH Wave Propagation in Weld. We chose the welded model to study the refractive characteristics of the $\mathrm{SH}$ wave. First, two steel plates $800 \times 600 \times 6 \mathrm{~mm}^{3}$ are given. Welding along the width direction was carried out to obtain a $600 \mathrm{~mm}$ long weld with a width of $10 \mathrm{~mm}$. In order to match the weld model with the steel plate model in the upper section, we grind the weld to get zero weld height. Material parameters $[34,35]$ of steel plates and weld seams are given in Table 2.

Table 2 shows the different material parameters of the welded structure and steel plate. The $\mathrm{SH}$ wave refracts at the interface of the welded structure and steel plate. At the interface, the micromodulus of the material needs to be recalculated, which increases the calculation of the welded structure and makes the SH wave propagation more complicated. The same load is applied to the same position as the steel plate in Section 3.2, and the sensors are arranged in the same manner, as shown in Figure 6.

In Figure 6, sensor $\mathrm{E}$ is located in the middle of the steel plate and on the $\mathrm{BC}$ line $30 \mathrm{~mm}$ from point $\mathrm{B}$; sensor $\mathrm{F}$ is $10 \mathrm{~mm}$ horizontal from the weld and $30 \mathrm{~mm}$ vertical from the edge of the steel plate; sensor $\mathrm{G}$ is $20 \mathrm{~mm}$ horizontal from the weld and $30 \mathrm{~mm}$ vertical from the edge of the steel plate; sensor $\mathrm{H}$ is $30 \mathrm{~mm}$ horizontal from the weld and $30 \mathrm{~mm}$ from the vertical edge of the steel plate.

The SH-guided wave is numerically simulated by the peridynamic method to study the propagation of the $\mathrm{SH}$ wave in the weld seam. The force load (29) in direction $x_{3}$ is applied on the surface, where point D is located. This will then generate $\mathrm{SH}$ that propagates along the weld seam with the vibration in direction $x_{3}$. The iteration step is $10^{-7} \mathrm{~s}$, and the simulation time is $1 \mathrm{~ms}$. The peridynamic subdivision is $h=2 \mathrm{~mm}$, the weight function is $\delta=3.1 \mathrm{~h}$, and the 
TABLE 2: Material parameters of the steel plate and weld seam.

\begin{tabular}{lcccc}
\hline & Elastic modulus $(\mathrm{GPa})$ & Poisson ratio & Density $\left(\mathrm{kg} / \mathrm{m}^{3}\right)$ & SH velocity $(\mathrm{m} / \mathrm{s})$ \\
\hline Steel plate & 210 & 0.30 & 7932 & 3191.04 \\
Weld seam & 192 & 0.33 & 7900 & 3022.71 \\
\hline
\end{tabular}

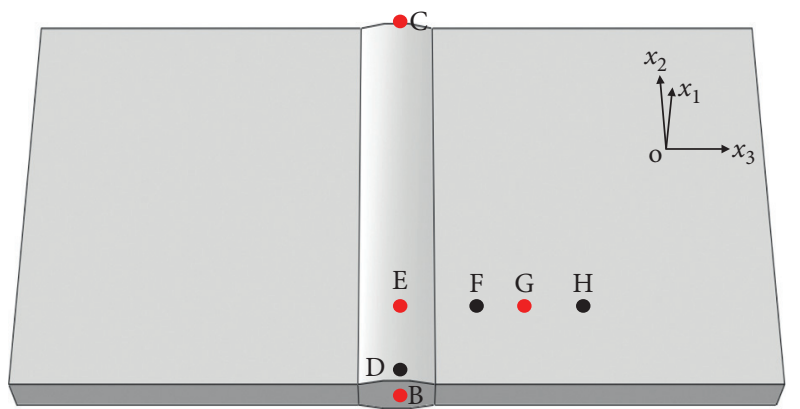

Figure 6: Sensor and load of the welded structure.

horizon radius is $\delta=3.1 \mathrm{~h}$. The signal in the sensor is obtained, as shown in Figure 7.

Figure 7 shows that the SH-guided waves propagated in the welded structure and steel plate are basically the same under the same conditions. In Figure 7, the signal in sensor E is stronger than that in Figure $3(E)$. This proves that the $\mathrm{SH}$ reflects between the weld seam and steel plate due to the slight difference of material parameters. This leads to more SH energy trapping in the weld seam $[36,37]$. The peak value of signal 3 in the sensor is selected to further study the propagation of the $\mathrm{SH}$ wave. Its data are presented in Table 3.

Table 3 shows that the peak time of signal 3 is gradually delayed with the sensor away from the weld seam, and there is an obvious decrease in the peak value of the signal. Compared with the peak value of signal 3 on sensor $E$ in Table 1, we see that the peak of the welded seam is larger than that of the steel plate structure, which is due to the reflection between the weld seam and steel plate, resulting in a large amount of energy trapped in the weld seam. By comparing the peak time of signal 3 , we see that signal 3 in the welded structure appears later than the steel structure. This is because the SH wave velocity of the weld seam is smaller than that of the steel plate. Compared with the data in Table 1, we see that the peak of signal 3 on sensors $F$ and $G$ is larger, and that on sensor $\mathrm{H}$ is smaller; this proves that the energy of the $\mathrm{SH}$ waves is concentrated around the weld seam. Formula (37) shows that the radiation rate of the $\mathrm{SH}$-guided wave is $33.4 \%$, which is much lower than that on the steel plate structure $(50.8 \%)$. This further proves that most of the SH energy is trapped in the weld seam $[4,37,38]$.

4.3. Weld Experiments. In order to study the refraction and reflection characteristics of $\mathrm{SH}$ wave in the weld seam, the material parameters, the size of the steel plate and the welded mode, and the positions of the ultrasonic sensor in the experiment are the same as those in the simulation (Figure 6). The load is excited by the SH wave PZT sensor at point D. Electrical signals are collected by the PZT sensor at
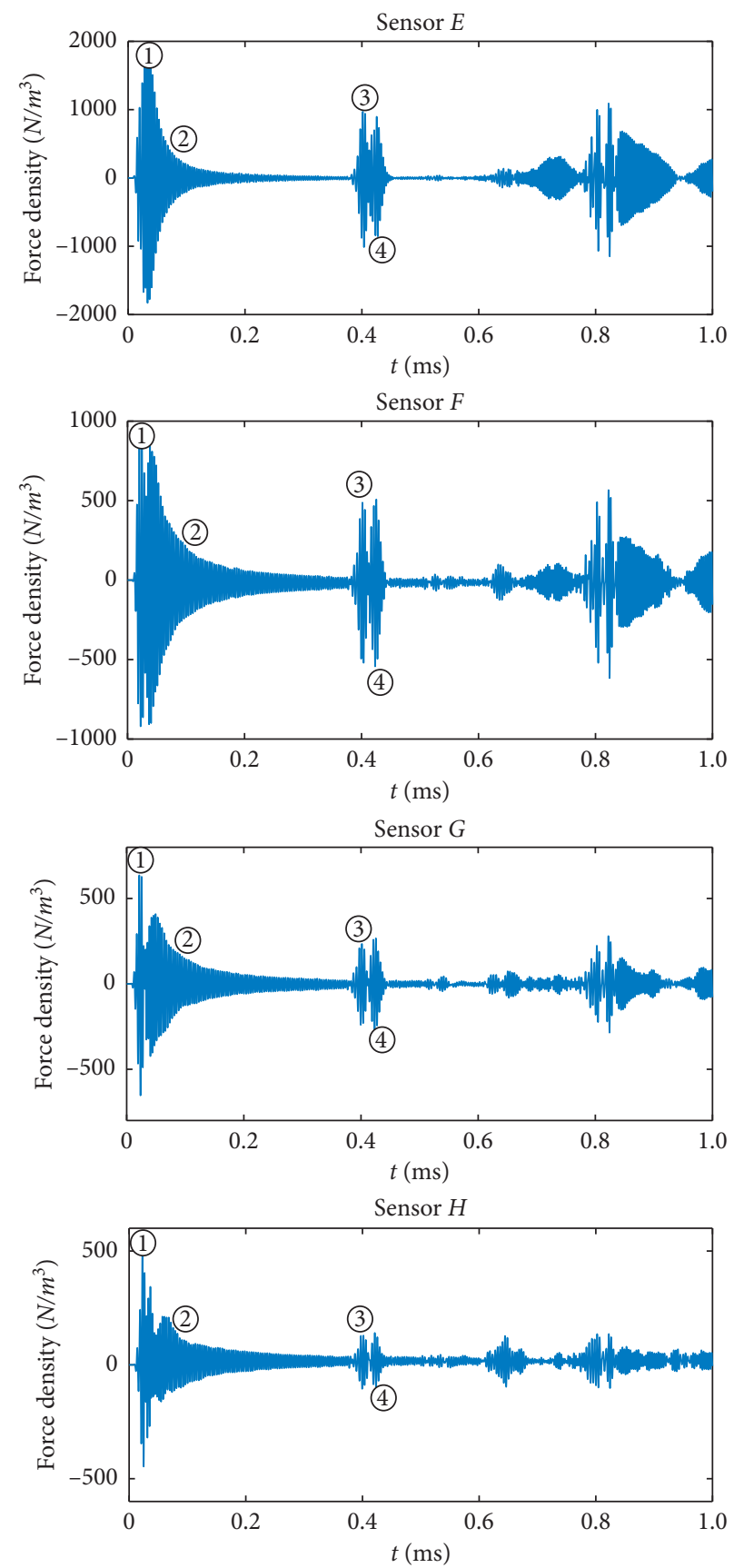

FIgURE 7: Sensor signal on the welded structure of numerical simulation.

the signal-receiving point $E$ and imaged by an oscilloscope. The resulting oscilloscope signal is shown in Figure 8.

The waveform signal is essentially the same in Figure 8 compared with Figures 4 and 7. Analysis of Figure 8 shows that there is $0.016 \mathrm{~V}$ clutter in the oscilloscope signal. 
TABLE 3: The data of signal 3 in the weld seam.

\begin{tabular}{lcccc}
\hline & Sensor E & Sensor F & Sensor G & Sensor H \\
\hline Time $(\mathrm{ms})$ & 0.4016 & 0.4016 & 0.4017 & 0.4017 \\
Amplitude $\left(\mathrm{N} / \mathrm{m}^{3}\right)$ & 970.7 & 487.9 & 234.5 & 129.0 \\
\hline
\end{tabular}

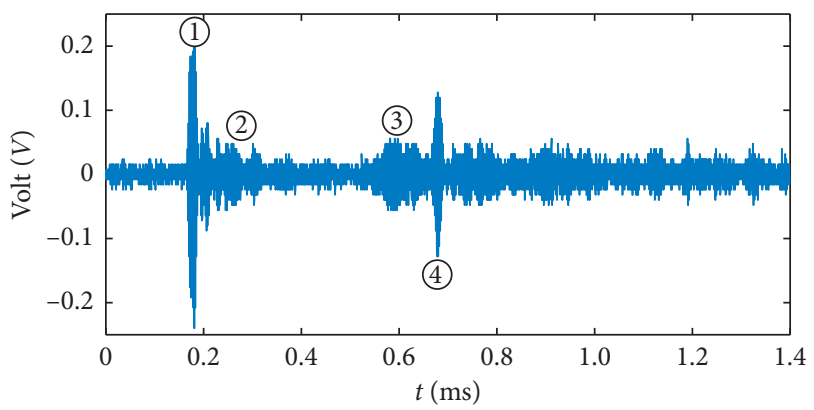

Figure 8: Oscilloscope signal on weld.

However, due to the randomness of the signal collected by the oscilloscope, the signal on the oscilloscope has a delay of $0.15 \mathrm{~ms}$. The peak value of signal 3 is $0.056 \mathrm{~V}$ at $0.581 \mathrm{~ms}$. The signal in Figures 3, 4 and 7 suggest that the formation of the weld-guided wave is mainly reflected by the upper and lower boundaries. The differences in material parameters between the steel plate and weld seam that generated the SH wave reflection between the interface aggravate the formation of the weld-guided wave [38].

\section{Conclusion}

In this paper, the internal force density of $\mathrm{SH}$ waves in peridynamic medium was analyzed based on the theory of peridynamics. The internal force density of the $\mathrm{SH}$ wave in peridynamic medium is only related to the shear modulus. The reflection characteristics of the $\mathrm{SH}$ wave are studied by the analysis of the material points near the reflection point in the virtual layer. The reflection characteristics of the $\mathrm{SH}$ wave in the steel plate are verified by the simulation and experiment, which are consistent with the traditional theory. The refraction characteristics of $\mathrm{SH}$ waves in the peridynamic medium are established by analyzing the material points at the refraction point, and the relationship between bonds and wave angle is established. We then compared simulation and experimental results of the steel plate with the welded structure and concluded that the SHguided wave in the weld seam is mainly formed by the reflection from the upper and lower surfaces, and the reflection between the weld seam and the steel plate only aggravates the formation of $\mathrm{SH}$-guided waves in the weld seam.

\section{Data Availability}

The data used to support the findings of this study are available from the corresponding author upon request.

\section{Conflicts of Interest}

The authors declare that they have no conflicts of interest.

\section{Acknowledgments}

The work was supported by the National Natural Science Foundation of China (no. 51679112) and Postgraduate Research \& Practice Innovation Program of Jiangsu Province KYCX17_1764. The authors thank LetPub (http://www. letpub.com) for its linguistic assistance during the preparation of this manuscript.

\section{References}

[1] J. D. Achenbach, "Modeling for quantitative non-destructive evaluation," Ultrasonics, vol. 40, no. 1-8, pp. 1-10, 2002.

[2] A. Pau and F. Lanza di Scalea, "Nonlinear guided wave propagation in prestressed plates," The Journal of the Acoustical Society of America, vol. 137, no. 3s, pp. 1529-1540, 2015.

[3] A. Pau, D. V. Achillopoulou, and F. Vestroni, "Scattering of guided shear waves in plates with discontinuities," NDT \& $E$ International, vol. 84, pp. 67-75, 2016.

[4] Z. Fan, M. Castaings, M. J. S. Lowe, C. Biateau, and P. Fromme, "Feature-guided waves for monitoring adhesive shear modulus in bonded stiffeners," NDT \& E International, vol. 54, pp. 96-102, 2013.

[5] J. L. Rose, Ultrasonic Guided Waves in Solid Media, Cambridge University Press, Cambridge, UK, 2014.

[6] A. K. Singh and S. Guha, "Reflection of plane waves from the surface of a piezothermoelastic fiber-reinforced composite half-space," Mechanics of Advanced Materials and Structures, pp. 1-13, 2020.

[7] P. Singh, A. K. Singh, A. Chattopadhyay, and S. Guha, "Mathematical study on the reflection and refraction phenomena of three-dimensional plane waves in a structure with floating frozen layer," Applied Mathematics and Computation, vol. 386, p. 125488, 2020.

[8] S. Guha, A. K. Singh, and A. Das, "Analysis on different types of imperfect interfaces between two dissimilar piezothermoelastic half-spaces on reflection and refraction phenomenon of plane waves," Waves in Random and Complex Media, pp. 1-30, 2019.

[9] S. Guha and A. Kumar Singh, "Effects of initial stresses on reflection phenomenon of plane waves at the free surface of a rotating piezothermoelastic fiber-reinforced composite halfspace," International Journal of Mechanical Sciences, vol. 181, p. 105766, 2020.

[10] Y. Shaogan, Z. Junhui, X. Bing, S. Wei, and Z. Shiqiang, "A hybrid lumped parameters/finite element/boundary element model to predict the vibroacoustic characteristics of an axial piston pump," Shock and Vibration, vol. 2017, Article ID 3871989, 11 pages, 2017.

[11] Q. Zhang, X. Wang, L.-g. Tian, and D.-m. Huang, “Analysis of mechanical and acoustic emission characteristics of rock materials with double-hole defects based on particle flow code," Shock and Vibration, vol. 2018, Article ID 7065029, 11 pages, 2018.

[12] X. Zhongyuan and C. Wei, "Vibration analysis of plate with irregular cracks by differential quadrature finite element method," Shock and Vibration, vol. 2017, Article ID 2073453, 13 pages, 2017. 
[13] T. Kamas, V. Giurgiutiu, and B. Lin, "Quasi-Rayleigh waves in butt-welded thick steel plate," in Proceedings of the AIP Conference on 2015, Jatinangor, Indonesia, September 2015.

[14] A. Pau, D. Capecchi, and F. Vestroni, "Reciprocity principle for scattered fields from discontinuities in waveguides," Ultrasonics, vol. 55, pp. 85-91, 2015.

[15] Q. Liu, C. Zhang, and M. I. Todorovska, "Scattering of SH waves by a shallow rectangular cavity in an elastic half space," Soil Dynamics and Earthquake Engineering, vol. 90, pp. 147157, 2016.

[16] D. V. Achillopoulou and A. Pau, "Characterization of defects in plates using shear and Lamb waves," Procedia Engineering, vol. 199, pp. 2001-2007, 2017.

[17] Z. Fan, J. Zhang, H. Xu, and J. Cai, "Transmission of normal P-waves across a single joint based on model," Shock and Vibration, vol. 2019, Article ID 8240586, , 2019.

[18] S. A. Silling, "Reformulation of elasticity theory for discontinuities and long-range forces," Journal of the Mechanics and Physics of Solids, vol. 48, no. 1, pp. 175-209, 2000.

[19] S. A. Silling, M. Zimmermann, and R. Abeyaratne, "Deformation of a peridynamic bar," Journal of Elasticity, vol. 73, no. 1-3, pp. 173-190, 2003.

[20] M. Zimmermann, A Continuum Theory with Long-Range Forces for Solids, Massachusetts Institute of Technology, Cambridge, MA, USA, 2005.

[21] S. A. Silling, M. Epton, O. Weckner, J. Xu, and E. Askari, "Peridynamic states and constitutive modeling," Journal of Elasticity, vol. 88, no. 2, pp. 151-184, 2007.

[22] S. A. Silling, "Linearized theory of peridynamic states," Journal of Elasticity, vol. 99, no. 1, pp. 85-111, 2010.

[23] Z. P. Bažant, W. Luo, V. T. Chau, and M. A. Bessa, "Wave dispersion and basic concepts of peridynamics compared to classical nonlocal damage models," Journal of Applied Mechanics, vol. 83, 2016.

[24] S. A. Silling, "Solitary waves in a peridynamic elastic solid," Journal of the Mechanics and Physics of Solids, vol. 96, pp. 121-132, 2016.

[25] S. N. Butt, J. J. Timothy, and G. Meschke, "Wave dispersion and propagation in state-based peridynamics," Computational Mechanics, vol. 60, pp. 1-14, 2017.

[26] X. Zhang, Z. Xu, and Q. Yang, "Wave dispersion and propagation in linear peridynamic media," Shock and Vibration, vol. 2019, Article ID 9528978, 9 pages, 2019.

[27] E. Emmrich and O. Weckner, "The peridynamic equation and its spatial discretisation," Mathematical Modelling and Analysis, vol. 12, no. 1, pp. 17-27, 2007.

[28] S. A. Silling and R. B. Lehoucq, "Peridynamic theory of solid mechanics," Advances in Applied Mechanics, vol. 44, pp. 73$168,2010$.

[29] S. A. Silling, O. Weckner, E. Askari, and F. Bobaru, "Crack nucleation in a peridynamic solid," IUTAM Symposium on Dynamic Fracture and Fragmentation, vol. 162, pp. 219-227, 2010.

[30] E. Madenci and E. Oterkus, Peridynamic Theory and its Applications, Springer, Berlin, Germany, 2014.

[31] J. Achenbach, Wave Propagation in Elastic Solids, Elsevier, Amsterdam, Netherlands, 2012.

[32] F. Bobaru, J. T. Foster, P. H. Geubelle, and S. A. Silling, Handbook of Peridynamic Modeling, CRC Press, Boca Raton, FA, USA, 2016.

[33] X. Liu, W. Gu, J. Liu, Z. Wang, J. Xu, and T. Cao, "Investigation of the propagation characteristics of underwater shock waves in underwater drilling blasting," Shock and Vibration, p. 2018, Article ID 9483756, 2018.
[34] X. Zhang and Z. Xu, "Dispersion of an SH-guided wave in weld seam based on peridynamics theory," Mathematical Problems in Engineering, vol. 2020, Article ID 4802930, 9 pages, 2020.

[35] X.-L. Zhang, Z.-Y. Xu, T. Yao, Y.-X. Wang, and M.-Q. Wu, The Simulation of SH Guided Wave at Weld by Peridynamics Theory, 2019 IEEE Far East NDT New Technology and Application Forum, FENDT 2019, Institute of Electrical and Electronics Engineers Inc., Qingdao, China, 2019.

[36] Z. Fan and M. J. S. Lowe, "Interaction of weld-guided waves with defects," NDT \& E International, vol. 47, pp. 124-133, 2012.

[37] Z. Fan and M. J. Lowe, "Elastic waves guided by a welded joint in a plate," in Proceedings of the Royal Society of London A: Mathematical, Physical and Engineering Sciences, London, UK, August 2009.

[38] X. Zhang and Z. Xu, "Formation mechanism of SH guided wave in weld seam," Results in Physics, vol. 16, Article ID 102840, 2020. 